\title{
Enhanced 2D-DOA Estimation for Large Spacing Three-Parallel Uniform Linear Arrays
}

\author{
Dong Zhang $(\mathbb{D}$, Yongshun Zhang, and Cunqian Feng \\ Air and Missile Defense College, Air Force Engineering University, Xi'an, Shaanxi 710051, China \\ Correspondence should be addressed to Dong Zhang; zhangdongtougao@163.com
}

Received 15 September 2017; Revised 9 January 2018; Accepted 30 January 2018; Published 4 March 2018

Academic Editor: Elisa Giusti

Copyright (c) 2018 Dong Zhang et al. This is an open access article distributed under the Creative Commons Attribution License, which permits unrestricted use, distribution, and reproduction in any medium, provided the original work is properly cited.

\begin{abstract}
An enhanced two-dimensional direction of arrival (2D-DOA) estimation algorithm for large spacing three-parallel uniform linear arrays (ULAs) is proposed in this paper. Firstly, we use the propagator method (PM) to get the highly accurate but ambiguous estimation of directional cosine. Then, we use the relationship between the directional cosine to eliminate the ambiguity. This algorithm not only can make use of the elements of the three-parallel ULAs but also can utilize the connection between directional cosine to improve the estimation accuracy. Besides, it has satisfied estimation performance when the elevation angle is between $70^{\circ}$ and $90^{\circ}$ and it can automatically pair the estimated azimuth and elevation angles. Furthermore, it has low complexity without using any eigen value decomposition (EVD) or singular value decompostion (SVD) to the covariance matrix. Simulation results demonstrate the effectiveness of our proposed algorithm.
\end{abstract}

\section{Introduction}

Two-dimensional DOA estimation has attracted extensive attention due to its wide range of applications [1-4], and there are many algorithms been proposed for DOA estimation. Among these algorithms, the subspace-based algorithms, such as MUSIC and ESPRIT, have received a lot of attention for its accurate estimation performance $[5,6]$. However, the complexity of subspace-based algorithms is often too large because of the EVD or SVD. In [7], Marcos et al. put forward the wellknown propagator method (PM) algorithm for 1D-DOA estimation. It uses linear partitioning instead of any EVD or SVD to reduce complexity. Then, Chen et al. [8] extend it to the DOA estimation of noncircular signal. References [9-11] extend the PM algorithm to 2D direction estimation. But these algorithms have some drawbacks. Reference [9] requires angle search operations. Reference [10] may fail in practical situation when elevation angle is between $70^{\circ}$ and $90^{\circ}$. Reference [11] has worse estimation accuracy, because some element information is missing when calculating the propagation matrix (PMA). Based on this, Chen et al. [12] put forward an improved 2D angle estimation algorithm for threeparallel ULAs. It can solve all the problems mentioned in the above literature. But, it still has some shortcomings, that is, the array aperture is reduced because of the adoption of three parallel arrays with half wavelength spacing. And the estimation accuracy is reduced. References $[13,14]$ establish the "array of subarrays" idea and "cyclic ambiguity" idea to improve the estimation accuracy of 2D-DOA. According to this, we expanded the spacing between the ULA and proposed an enhanced 2D-DOA estimation algorithm. What needs to be stressed here is that our method is different from the method in $[13,14]$, although our algorithm and the method in $[13,14]$ both use the large array spacing to obtain high accuracy but ambiguous estimation. However, the principle of resolving ambiguity is different. References $[13,14]$ use coarse estimation without ambiguity or based on eigen space to resolve ambiguity. However, our algorithm uses the triangular relation between the three directional cosine to resolve the ambiguity. By doing this, we do not have to limit ourselves to using subarrays or eigen spaces to solve ambiguity problems. That is the main difference between our method and the method in $[13,14]$. And it is also the main innovation of this paper. The flow of our algorithm is as follows: Firstly, we use the method in [12] to obtain the estimation of three directional cosines. Because of the large spacing between array elements, the estimated directional 
cosine is high precision and ambiguous. Then, we use the triangle relationship between the three directional cosines to eliminate the ambiguity. Then, we can get the true 2D-DOA of targets. Simulation results show that it cannot only avoid the problem in [8-11] simultaneously but also has better estimation accuracy than algorithm of [12] because of adopting large aperture. And the complexity of the algorithm is comparable to that algorithm of [12].

Notations. Superscripts $(\cdot)^{*},(\cdot)^{T},(\cdot)^{\dagger},(\cdot)^{-1}$, and $(\cdot)^{H}$ denote complex conjugation, transpose, pseudo-inverse, inverse, and conjugate transpose, respectively. $\mathbf{I}_{K}$ is $K \times K$ identity matrix. $\operatorname{diag}[\cdot]$ denotes the diagonalization of the entity inside. $|\cdot|$ denotes take the absolute value of the element. $\lceil\cdot\rceil$ denotes round the element to the nearest integers less than or equal to the element. $\lfloor\cdot\rfloor$ denotes round the element to the nearest integers greater than or equal to the element. $E[\cdot]$ denotes the expectation operation. $\arg (\cdot)$ and $\operatorname{Re}(\cdot)$ denote the phase and the real part of a complex number separately.

\section{Problem Formulation}

As shown in Figure 1, assume that there are three-parallel ULAs, namely, $X, Y$, and $Z$. Array $X$ contains $N+1$ sensors. Array $Y$ and array $Z$ have $N$ sensors, respectively. The spacing between adjacent elements is $d_{x}$. The distance between array $X$ and array $Y$ is $d_{y}$, and the distance between array $X$ and array $Z$ is $d_{z} \cdot \lambda$ is the wavelength. It is assumed that $P$ far-field narrowband uncorrelated signals are incident onto the array. The elevation angle and azimuth angle of $i$ th target are $\theta_{i}$ and $\varphi_{i}$, respectively. Here, we assume that the range of $2 \mathrm{D}$ angle is the same with that of [12]. That is to say that the range value of $\theta_{i}$ is $[0,1 / 2 \pi]$ and the range value of $\varphi_{i}$ is $[-1 / 2 \pi, 1 / 2 \pi]$. Then, the output of the three ULAs can be expressed as follows:

$$
\begin{aligned}
& \mathbf{X}(t)=\mathbf{A}_{x} \mathbf{S}(t)+\mathbf{W}_{x}(t) \\
& \mathbf{Y}(t)=\mathbf{A}_{y} \boldsymbol{\Omega}_{y} \mathbf{S}(t)+\mathbf{W}_{y}(t) \\
& \mathbf{Z}(t)=\mathbf{A}_{y} \boldsymbol{\Omega}_{z} \mathbf{S}(t)+\mathbf{W}_{z}(t)
\end{aligned}
$$

where $\mathbf{A}_{x}=\left[\mathbf{a}_{x}\left(\theta_{1}, \varphi_{1}\right), \mathbf{a}_{x}\left(\theta_{2}, \varphi_{2}\right), \ldots, \mathbf{a}_{x}\left(\theta_{P}, \varphi_{P}\right)\right]$ and $\mathbf{a}_{x}\left(\theta_{i}\right.$, $\left.\varphi_{i}\right)=\left[1, e^{-j 2 \pi d_{x} \sin \theta_{\mathrm{i}} \sin \varphi_{\mathrm{i}} / \lambda}, \ldots, e^{-j 2 \pi N d_{x} \sin \theta_{\mathrm{i}} \sin \varphi_{\mathrm{i}} / \lambda}\right] \cdot \mathbf{A}_{y}$ contains the first $\mathrm{N}$ row of $\mathbf{A}_{x} \cdot \boldsymbol{\Omega}_{y}=\operatorname{diag}\left(\left[e^{-j 2 \pi d_{y} \cos \theta_{1} / \lambda}, e^{-j 2 \pi d_{y} \cos \theta_{2} / \lambda}\right.\right.$, $\left.\left.\ldots, e^{-j 2 \pi d_{y} \cos \theta_{p} / \lambda}\right]\right) \quad$ and $\quad \boldsymbol{\Omega}_{z}=\operatorname{diag}\left(\left[e^{-j 2 \pi d_{z} \sin \theta_{1} \cos \varphi_{1} / \lambda}\right.\right.$, $\left.\left.e^{-j 2 \pi d_{z} \sin \theta_{2} \cos \varphi_{2} / \lambda}, \ldots, e^{-j 2 \pi d_{z} \sin \theta_{P} \cos \varphi_{P} / \lambda}\right]\right) . \mathbf{W}_{x}(t), \mathbf{W}_{y}(t)$, and $\mathbf{W}_{z}(t)$ are assumed to be Gaussian white noise vectors whose mean value is zero and variance is $\sigma^{2}$. Then a new vector $\mathbf{W}(t)=\left[\mathbf{X}(t)^{T} \mathbf{Y}(t)^{T} \mathbf{Z}(t)^{T}\right]^{T}$, with $\mathrm{L}$ snapshots, and $\mathbf{W}=[\mathbf{W}(1), \mathbf{W}(2), \ldots, \mathbf{W}(L)]$ can be represented as

$$
\mathbf{W}=\left[\begin{array}{c}
\mathbf{X} \\
\mathbf{Y} \\
\mathbf{Z}
\end{array}\right]=\left[\begin{array}{c}
\mathbf{A}_{x} \\
\mathbf{A}_{y} \boldsymbol{\Omega}_{y} \\
\mathbf{A}_{y} \boldsymbol{\Omega}_{z}
\end{array}\right] \mathbf{S}+\left[\begin{array}{c}
\mathbf{W}_{x} \\
\mathbf{W}_{y} \\
\mathbf{W}_{z}
\end{array}\right]=\mathbf{A S}+\mathbf{N}
$$

where $\mathbf{A}=\left[\left(\mathbf{A}_{x}\right)^{T}\left(\mathbf{A}_{y} \boldsymbol{\Omega}_{y}\right)^{T}\left(\mathbf{A}_{y} \boldsymbol{\Omega}_{z}\right)^{T}\right]^{T}, \mathbf{S}=[S(1), S(2), \ldots, S$ $(L)] \in C^{P \times L}$, and $\mathbf{N}=\left[\left(\mathbf{W}_{x}\right)^{T}\left(\mathbf{W}_{y}\right)^{T}\left(\mathbf{W}_{z}\right)^{T}\right]^{T} \in C^{(3 N+1) \times L}$.

\section{Enhanced 2D-DOA Estimation}

3.1. Highly Accurate but Ambiguous Estimation of Directional Cosine. Assuming that $d_{x}=d_{y}=d=\lambda / 2, d_{z}=K d$, where $K$ is a positive integer and $K>1$. The difference between our model and the model in [12] is that the spacing between the ULA is larger than half wavelength. The large spacing will lead to ambiguity of the directional cosine, but it would not affect the estimation method for them. So the first several steps of our algorithm is same with the algorithm of [12]. We abbreviate the first several procedures as follows:

Step 1. Compute the covariance matrix of $\mathbf{W}(t)$ by $\mathbf{R}_{\mathbf{W}}=$ $E\left[\mathbf{W} \mathbf{W}^{H}\right]$.

Step 2. Divide $\mathbf{R}_{\mathbf{W}}$ into two parts, that is, $\mathbf{R}_{\mathbf{W}}=\left[\mathbf{R}_{\mathbf{W} 1} \mathbf{R}_{\mathbf{W} 2}\right]$, where $\mathbf{R}_{\mathbf{W} 1} \in C^{(3 N+1) \times P}$ and $\mathbf{R}_{\mathbf{W} 2} \in C^{(3 N+1) \times(3 N+1-P)}$. Compute the propagator matrix (PMA) $\mathbf{P}$ by $\widehat{\mathbf{P}}=\left(\mathbf{R}_{\mathrm{W} 1}^{H} \mathbf{R}_{\mathbf{W} 1}\right)^{-1} \mathbf{R}_{\mathbf{W} 1}^{H} \mathbf{R}_{\mathbf{W} 2}$ and get the extended PMA by $\mathbf{P}_{e}=\left[\mathbf{I}_{P}^{H} \widehat{\mathbf{P}}\right]=$ $[\underbrace{\mathbf{P}_{x}^{T}}_{P \times(N+1)} \underbrace{\mathbf{P}_{y}^{T}}_{P \times N} \underbrace{\mathbf{P}_{z}^{T}}_{P \times N}]^{T}$

Step 3. Implement EVD on $\boldsymbol{\Psi}_{z}=\mathbf{P}_{x 1}^{+} \mathbf{P}_{z}=\mathbf{A}_{1} \boldsymbol{\Omega}_{z} \mathbf{A}_{1}{ }^{-1}$ to get eigenvalues $\widehat{\beta}_{i}$ and eigenvectors $\mathbf{A}_{1}^{\prime}$. They correspond to the diagonal elements of $\boldsymbol{\Omega}_{z}$ and the estimation of $\mathbf{A}_{1}$, separately. And $\mathbf{P}_{x 1}^{+}$is the first $N$ rows of $\mathbf{P}_{x}$.

Step 4. Let $\mathbf{B}_{1}=\mathbf{P}_{x 1} \mathbf{A}_{1}^{\prime}, \mathbf{B}_{2}=\mathbf{P}_{y} \mathbf{A}_{1}^{\prime}$. Attain $\widehat{\alpha}_{i}$ by extracting the $i$ th diagonal elements of $\mathbf{B}_{1}^{+} \mathbf{B}_{2}$. Similarly, let $\mathbf{C}_{1}=$ $\left[\mathbf{P}_{x 1}^{T} \mathbf{P}_{y 1}^{T} \mathbf{P}_{z 1}^{T}\right]^{T} \mathbf{A}_{1}^{\prime}$ and $\mathbf{C}_{2}=\left[\mathbf{P}_{x 2}^{T} \mathbf{P}_{y 2}^{T} \mathbf{P}_{z 2}^{T}\right]^{T} \mathbf{A}_{1}^{\prime}$. Attain $\hat{\gamma}_{i}$ by extracting the $i$ th diagonal elements of $\mathbf{C}_{1}^{+} \mathbf{C}_{2}$. And $\mathbf{P}_{y 1}$ and $\mathbf{P}_{z 1}$ are the first $N-1$ rows of $\mathbf{P}_{y}$ and $\mathbf{P}_{z} \cdot \mathbf{P}_{y 2}$ and $\mathbf{P}_{z 2}$ are the last $N-1$ rows of $\mathbf{P}_{y}$ and $\mathbf{P}_{z} \cdot \mathbf{P}_{x 2}$ represents the last $N$ rows of $\mathbf{P}_{x}$.

Until now, we get the paired estimation of $\widehat{\alpha}_{i}, \widehat{\beta}_{i}$, and $\widehat{\gamma}_{i}$ which represent $\cos \theta_{i}, \sin \theta_{i} \cos \varphi_{i}$, and $\sin \theta_{i} \sin \varphi_{i}$, respectively. Because $d_{z}$ is larger than half wavelength, so the $\widehat{\beta}_{i}$ is high accuracy but ambiguous. And all the ambiguous values are listed as follows:

$$
\widehat{\beta}_{i}^{(k)}=\widehat{\beta}_{i}+k \frac{\lambda}{d_{z}}, \quad\left\lceil\left(-1-\widehat{\beta}_{i}\right) \frac{d_{z}}{\lambda}\right\rceil \leq k \leq\left\lfloor\left(1-\widehat{\beta}_{i}\right) \frac{d_{z}}{\lambda}\right\rfloor,
$$

where $i=1, \ldots, P$.

3.2. Highly Accurate Estimation of 2D-DOA. Before estimating the 2D-DOA, we need to eliminate the ambiguity first. Note that there exists a triangle relationship between these directional cosines:

$$
\left(\cos \theta_{i}\right)^{2}+\left(\sin \theta_{i} \cos \varphi_{i}\right)^{2}+\left(\sin \theta_{i} \sin \varphi_{i}\right)^{2}=1 .
$$




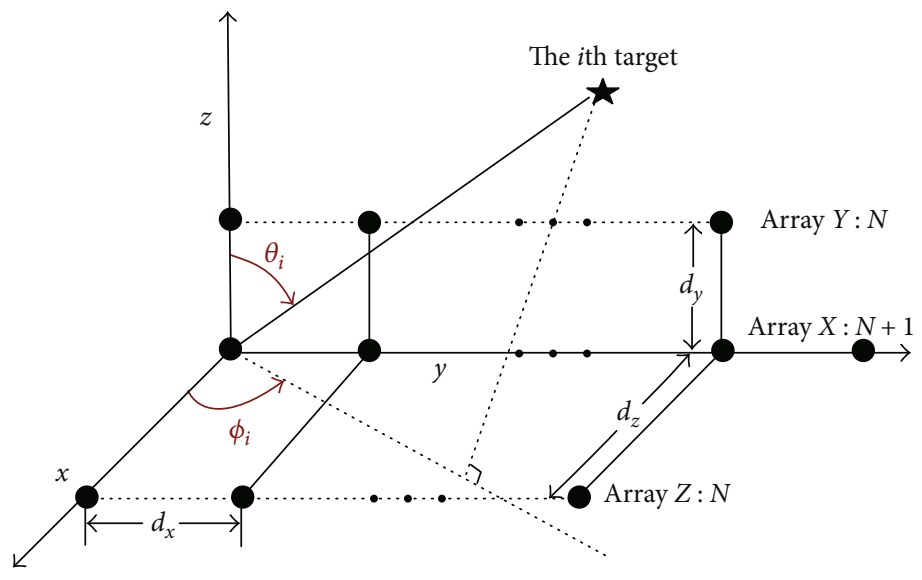

FIgURE 1: Sketch map of array structure.

And the true directional cosine of targets satisfies this equation. So, we can use this equation to select the true directional cosine from the ambiguous directional cosine.

But because there are square terms in the constraint, the mirror image of the true value also satisfies this equation. If the mirror image is exactly equal to an ambiguous value of the true value, we may make mistakes and treat the mirror image as the true value. Here, we set the range value of $\theta$ and $\varphi$ as $[0,1 / 2 \pi]$ and $[-1 / 2 \pi, 1 / 2 \pi]$, separately. So the true value of $\sin \theta_{i} \cos \varphi_{i}$ is limited in the range of $[0,1]$. And the mirror image can be avoided.

And the procedure of eliminating ambiguity can be got as follows:

Step 1. Get all the ambiguous value of $\sin \theta_{i} \cos \varphi_{i}$ by

$$
\widehat{\beta}_{i}^{(k)}=\widehat{\beta}_{i}+k \frac{\lambda}{d_{z}}, \quad\left\lceil\left(0-\widehat{\beta}_{i}\right) \frac{d_{z}}{\lambda}\right\rceil \leq k \leq\left\lfloor\left(1-\widehat{\beta}_{i}\right) \frac{d_{z}}{\lambda}\right\rfloor .
$$

Step 2. Find the true value by

$$
\widehat{\beta}_{i}^{(\widehat{k})}=\widehat{\beta}_{i}+\widehat{k} \frac{\lambda}{d_{z}}, \quad \widehat{k}=\underset{k}{\arg \min }\left|\alpha_{i}^{2}+\left[\widehat{\beta}_{i}^{(k)}\right]^{2}+\gamma_{i}^{2}-1\right| .
$$

Lastly, we can get the 2D-DOA estimation for each source by

$$
\begin{aligned}
& \widehat{\varphi}_{i}=\tan ^{-1}\left[\frac{\arg \left(\widehat{\gamma}_{i}\right)}{\arg \left(\hat{\beta}_{i}^{(\widehat{k})}\right)}\right], \\
& \widehat{\theta}_{i}=\tan ^{-1}\left[\frac{\arg \left(\hat{\beta}_{i}^{(\hat{k})}\right)}{\arg \left(\widehat{\alpha}_{i}\right) \cos \widehat{\varphi}_{i}}\right] .
\end{aligned}
$$

3.3. Algorithm Analysis. Here, the analysis of the complexity, estimation performance, and some notices of our algorithm are as follows:
(1) Our algorithm has an additional ambiguity elimination operation compared with the algorithm of [12]. And the complexity of ambiguity elimination is far less than the complexity of constructing the covariance. Thus, we can say that the algorithm has comparable computational complexity with the algorithm of [12].

(2) Because our array aperture is larger than that in [12], so our proposed algorithm can obtain a better estimation accuracy.

(3) Note that here we expand the spacing between the $X$-axis and $Z$-axis. Similarly, we can also expand the spacing between the $X$-axis and $Y$-axis or simultaneously expand the spacing in the $X$-axis, $Y$-axis, and $Z$-axis. Then, we can get high accuracy but ambiguous estimation of $\sin \theta_{i} \sin \varphi_{i}$ and $\cos \theta_{i}$, respectively. And we can still use (4) to eliminate the ambiguity. But considering the mirror image problem, the range of angle needs to be adjusted accordingly.

(4) Because the first several steps of our algorithm are the same with those of [12], so our algorithm inherits the merits of algorithm of [12]. For example, (1) it can take full advantage of the elements of the threeparallel ULAs to estimate the PMA; (2) it has satisfied estimation accuracy in actual mobile elevation angles; (3) it can automatically pair the estimated 2D-DOA; (4) it has low complexity without using any EVD or SVD.

\section{Computer Simulations}

Here, we will conduct several simulation experiments to test the performance of our proposed method. The 2D-DOA of two uncorrelated targets are $\left(\theta_{1}, \varphi_{1}\right)=\left(35^{\circ}, 40^{\circ}\right)$ and $\left(\theta_{2}, \varphi_{2}\right)=\left(80^{\circ}, 75^{\circ}\right)$, separately. $N$ is set to 6 . So the total number of array elements is 19 . The spacing between $X$-axis and $Z$-axis is set to $d_{z}=3 d$. The other spacing is set to $d_{x}=d_{y}=d$. 

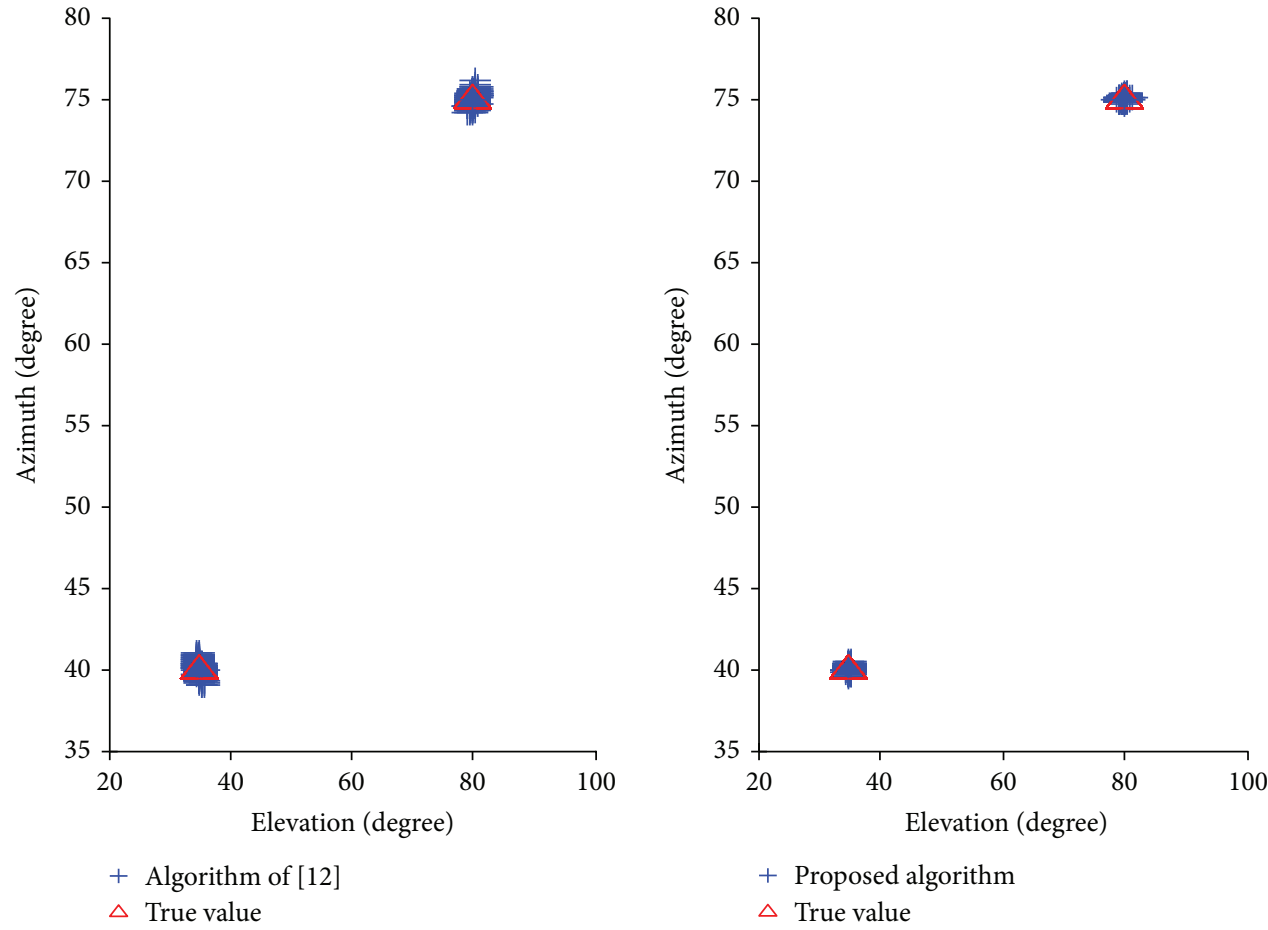

Figure 2: 2D-DOA scattergram at $\mathrm{SNR}=10 \mathrm{~dB}$.

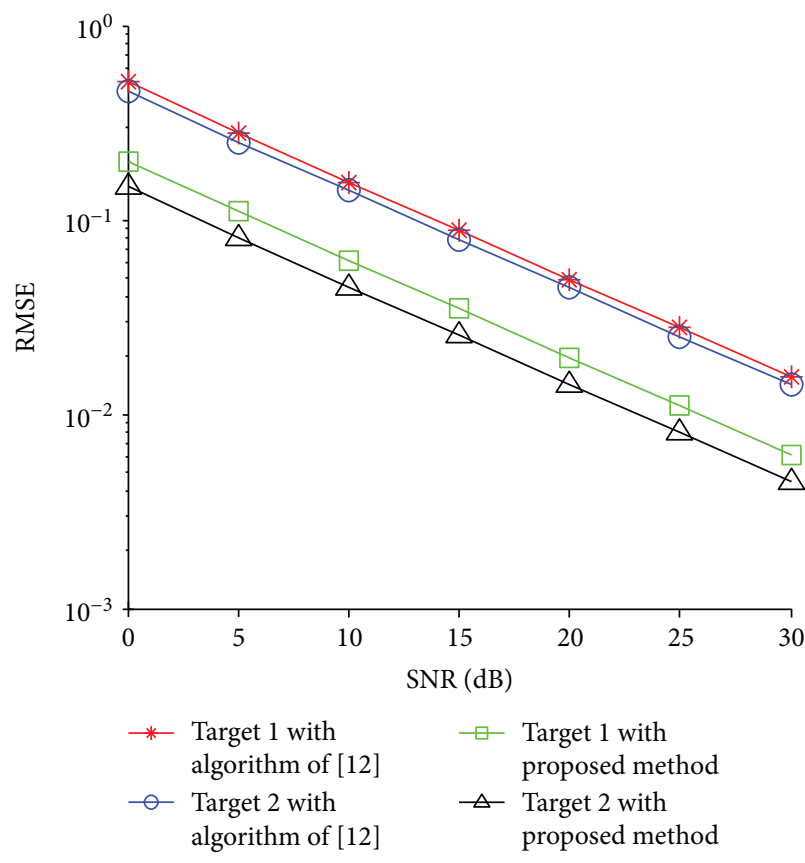

(a) RMSE of azimuth estimation

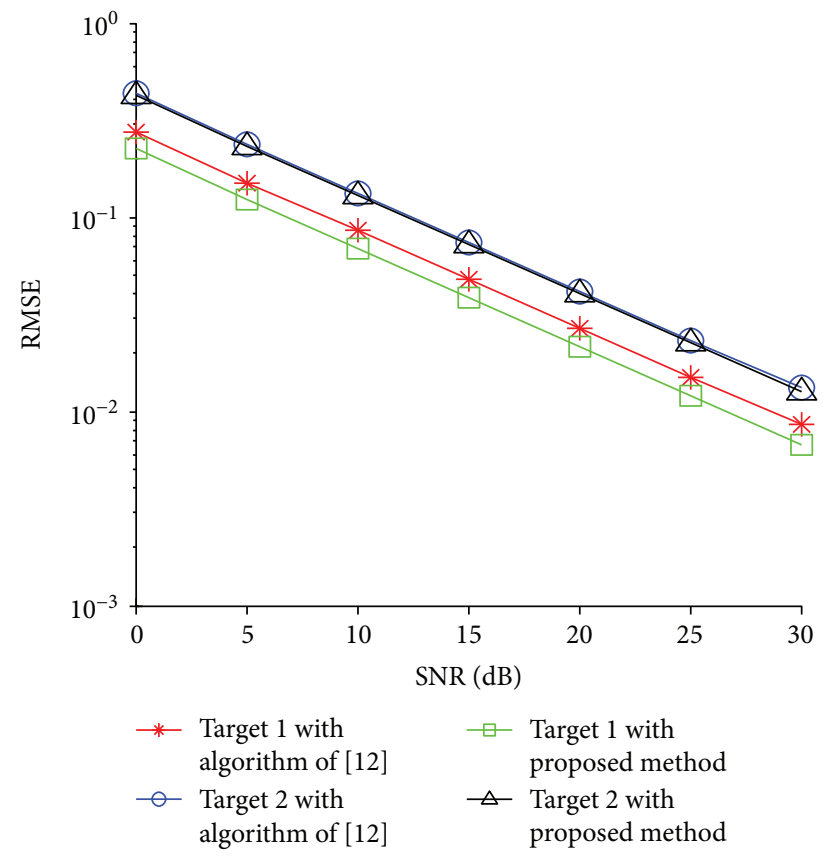

(b) RMSE of elevation estimation

FIgURE 3: RMSE contrast of two algorithms with different SNR.

In the first simulation, Figure 2 shows the scattergram of 300 times 2D-DOA estimate results by our algorithm and the algorithm of [12]. The snapshot is set to 50 . The $\mathrm{SNR}=10 \mathrm{~dB}$. As we can see, both the two algorithms can clearly observe the two targets. But our algorithm has better statistical properties. That is because we make better use of the information between ULAs.
In the second simulation, we compare the estimation performance of the two algorithms under different SNR which vary from $0 \mathrm{~dB}$ to $30 \mathrm{~dB}$. The snapshot is set to 300.200 times Monte Carlo simulations are conducted under each SNR. As illustrated in Figure 3, the RMSE curve of azimuth estimated by our algorithm is better than that estimated by the algorithm of [12]. The elevation estimation performance of our 


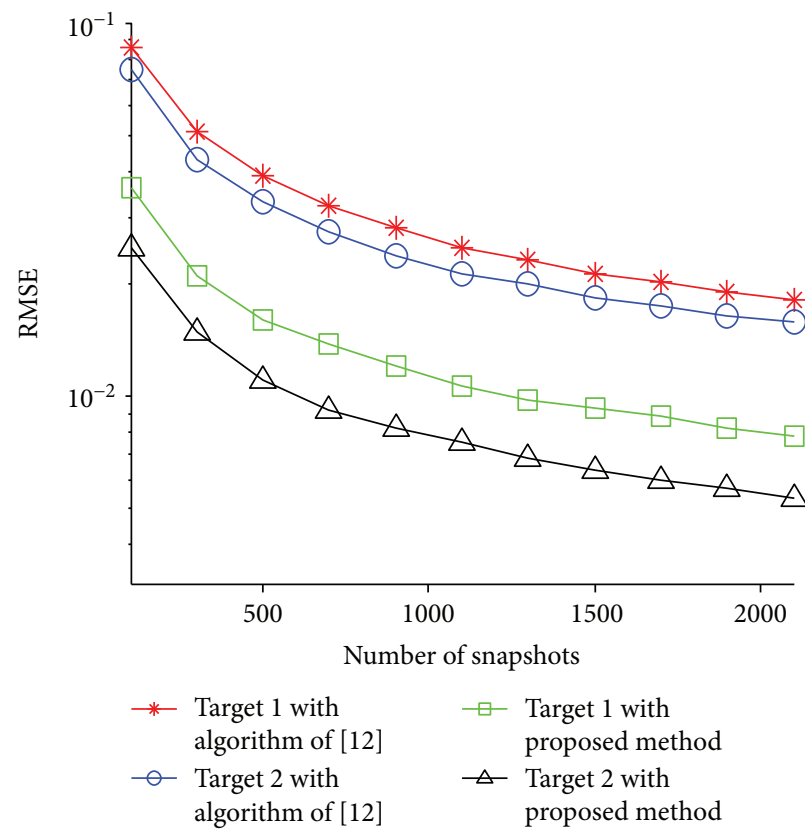

(a) RMSE of azimuth estimation

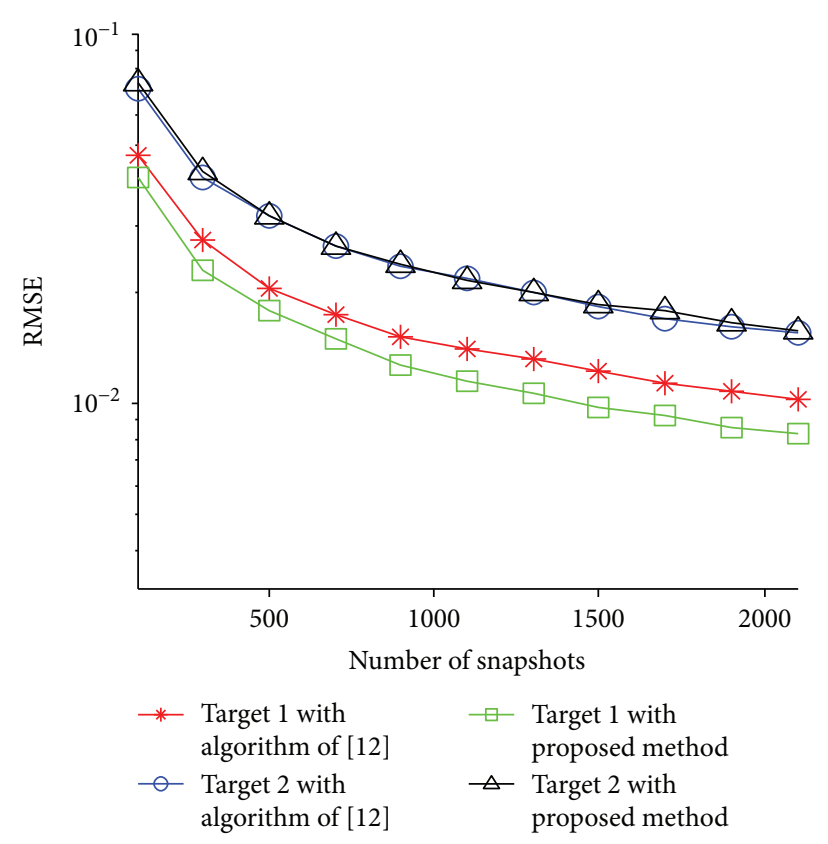

(b) RMSE of elevation estimation

FIGURE 4: RMSE contrast of two algorithms with different snapshots.

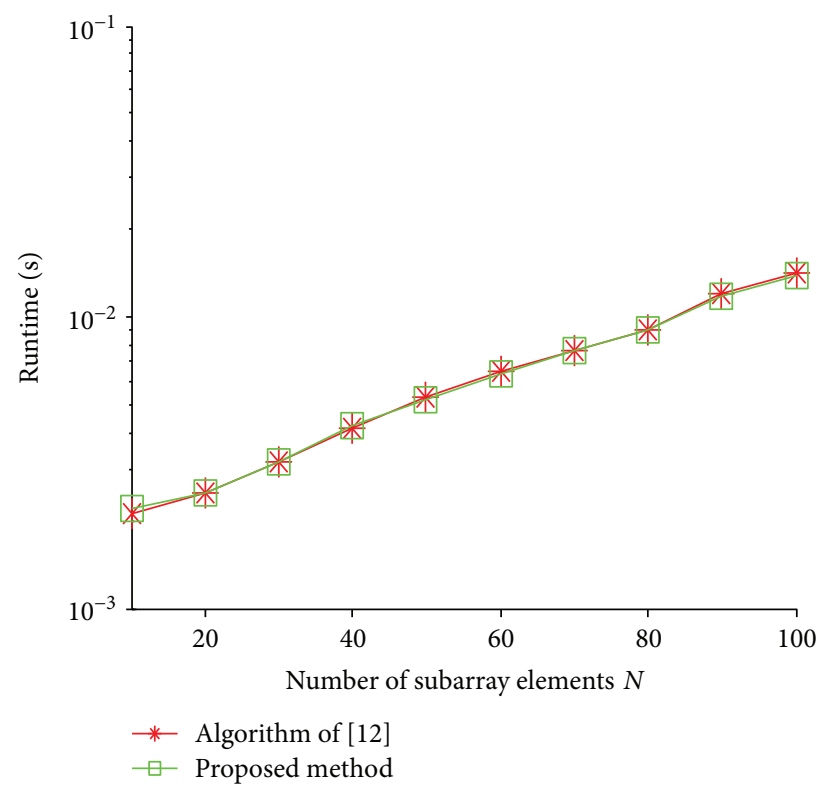

(a) Runtime versus element number

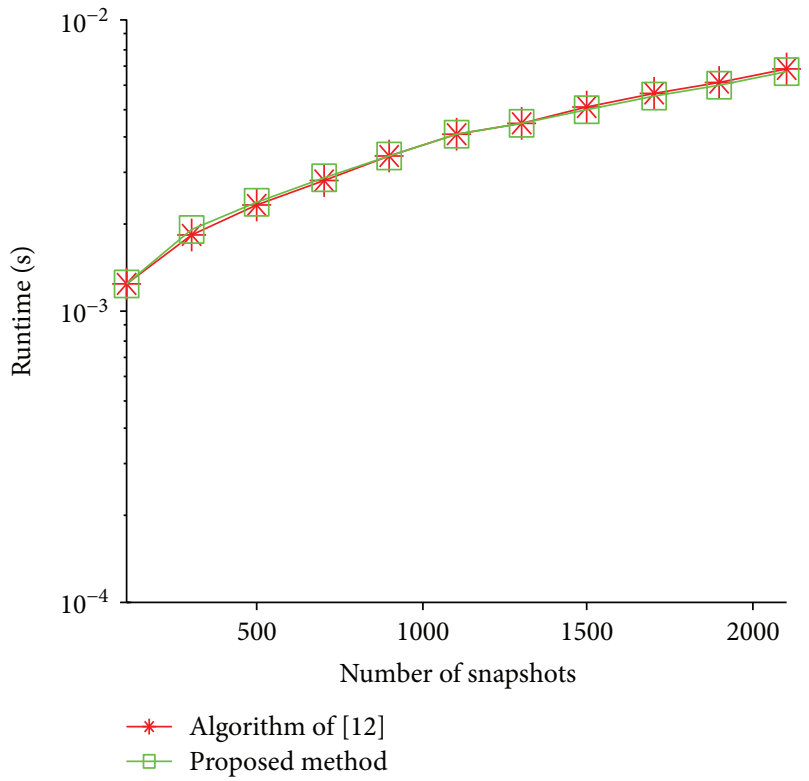

(b) Runtime versus number of snapshots

FIGURE 5: Runtime comparison of two algorithms.

algorithm is comparable with that estimated by the algorithm of [12]. The reason is that when estimating azimuth angle, the equivalent array aperture of our algorithm is larger than that of [12]. While when estimating elevation angle, the equivalent array aperture of our algorithm is equal with that of [12]. Note that if we want to improve the estimation accuracy of elevation angle, we can expand $d_{y}$ instead of expanding $d_{z}$.

In the third simulation, we compare the estimation performance of the two algorithms under different snapshots which vary from 100 to 2100 . The SNR is set to $20 \mathrm{~dB}$. 1000 times Monte Carlo simulations are conducted under each snapshot. As illustrated in Figure 4, the RMSE curve of azimuth estimated by our algorithm is better than that estimated by the algorithm of [12]. The elevation estimation performance of the two algorithms is similar. The reason is the same with the previous one. From the second and third simulations, we can also see that our algorithm has satisfied estimation performance when elevation is between $70^{\circ}$ and $90^{\circ}$. 


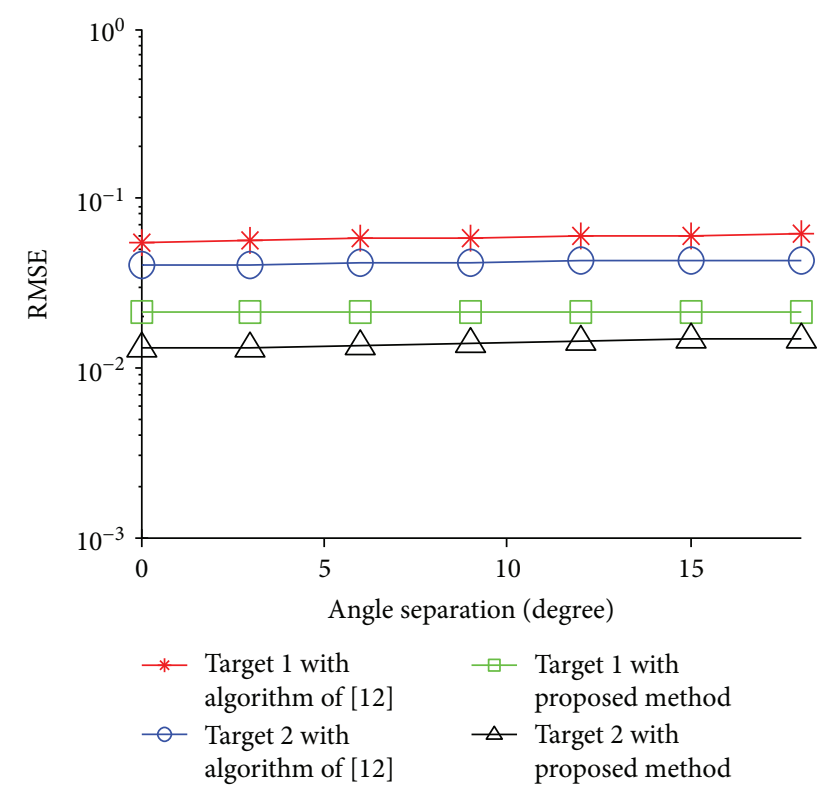

FIGURE 6: RMSE of azimuth estimation versus angular separation.

In the fourth simulation, we compare the complexity of our algorithm and that of [12]. We choose the runtime as the evaluation criteria. Two kinds of comparison were conducted. In the first comparison, the number of elements in subarray, that is, $N$, is set to vary from 10 to 100 and the snapshot is set to 300. The result is shown in Figure 5(a). In the second comparison, the snapshot is set to vary from 100 to 2100 . The number of elements in subarray is set to 20. And the result is shown in Figure 5(b). From the two figures, we can see that the runtime of our algorithm is similar with that of [12] which has shown the efficiency of our proposed algorithm.

In the fifth simulation, we compare the azimuth estimation performance of the two algorithms with different separation of angle. The 2D-DOA of two targets are $\left(\theta_{1}, \varphi_{1}\right)=\left(30^{\circ}, 60^{\circ}+\Delta \varphi_{1}\right)$ and $\left(\theta_{2}, \varphi_{2}\right)=\left(50^{\circ}, 70^{\circ}+\Delta \varphi_{2}\right)$, respectively. Both $\Delta \varphi_{1}$ and $\Delta \varphi_{2}$ vary from $0^{\circ}$ to $18^{\circ}$. The SNR is equal to $20 \mathrm{~dB}$. The snapshot is set to 500.200 times Monte Carlo simulations are conducted under each separation of angle. Here, the "angle separation" denotes $\Delta \varphi_{1}$ and $\Delta \varphi_{2}$ which vary from $0^{\circ}$ to $18^{\circ}$. It is different from the common definition of "angle separation." In fact, the separation angle of the two targets is fixed, what changes is the azimuth angle of the two targets. The purpose of doing this is to make the azimuth angle traverse all angles between $70^{\circ}$ and $88^{\circ}$ to prove the effectiveness of the algorithm in practical application. The result is shown in Figure 6. We can see that the performance of our proposed algorithm is better than that of the algorithm of [12].

In the sixth simulation, we compare the elevation estimation performance of the two algorithms with different separation of angle. Here, we set $d_{x}=d_{z}=d$ and $d_{y}=3 d$. The 2D-DOA of the two targets are $\left(\theta_{1}, \varphi_{1}\right)=\left(60^{\circ}+\Delta \theta_{1}\right.$, $\left.30^{\circ}\right)$ and $\left(\theta_{2}, \varphi_{2}\right)=\left(70^{\circ}+\Delta \theta_{2}, 50^{\circ}\right)$, respectively. Both $\Delta \theta_{1}$ and $\Delta \theta_{2}$ vary from $0^{\circ}$ to $18^{\circ}$. The other parameters are the same with the fifth simulation. The result is

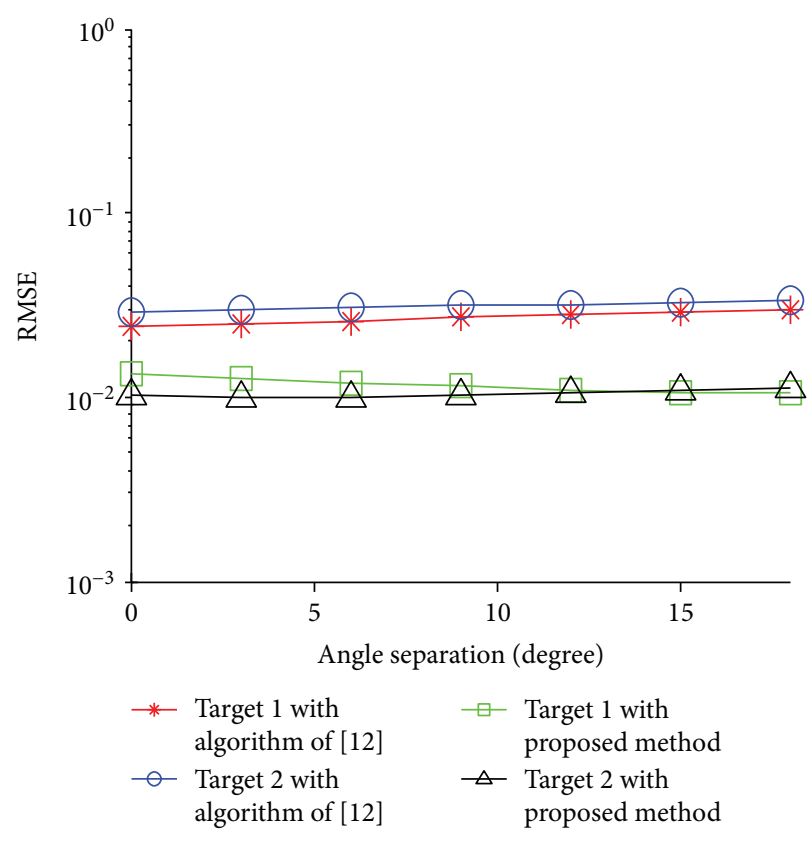

FIgURE 7: RMSE of elevation estimation versus angular separation.

shown in Figure 7. We can see that the performance of our proposed algorithm is better than that of the algorithm of [12].

\section{Conclusions}

An enhanced 2D-DOA estimation algorithm with threeparallel large spacing ULAs is proposed in this paper. It cannot only make use of the elements of the three-parallel ULAs to estimate the PMA but it can also utilize the information between three-parallel ULAs to improve the estimation accuracy. Besides, it has satisfied estimation performance when the elevation angle is between $70^{\circ}$ and $90^{\circ}$ and it can automatically pair the estimated 2D-DOA. Furthermore, it has low complexity without using any EVD or SVD to the covariance matrix. Simulations proved the effectiveness of the proposed algorithm.

\section{Conflicts of Interest}

The authors declare that they have no conflicts of interest.

\section{References}

[1] J. Li and R. T. Compton, "Two-dimensional angle and polarization estimation using the ESPRIT algorithm," IEEE Transactions on Antennas and Propagation, vol. 40, no. 5, pp. 550-555, 1992.

[2] T.-H. Liu and J. M. Mendel, "Azimuth and elevation direction finding using arbitrary array geometries," IEEE Transactions on Signal Processing, vol. 46, no. 7, pp. 2061-2065, 1998.

[3] Z. Ye and C. Liu, "2-D DOA estimation in the presence of mutual coupling," IEEE Transactions on Antennas and Propagation, vol. 56, no. 10, pp. 3150-3158, 2008.

[4] M. D. Zoltowski and K. T. Wong, "ESPRIT-based 2-D direction finding with a sparse uniform array of electromagnetic 
vector sensors," IEEE Transactions on Signal Processing, vol. 48, no. 8, pp. 2195-2204, 2000.

[5] R. Roy and T. Kailath, "ESPRIT-estimation of signal parameters via rotational invariance techniques," IEEE Transactions on Acoustics, Speech, and Signal Processing, vol. 37, no. 7, pp. 984-995, 1989.

[6] R. O. Schmidt, "Multiple emitter location and signal parameter estimation," in Proceedings RADC Spectrum Estimation Workshop, pp. 243-258, Griffiss AFB, Rome, NY, USA, 1979.

[7] S. Marcos, A. Marsal, and M. Benidir, "The propagator method for source bearing estimation," Signal Processing, vol. 42, no. 2, pp. 121-138, 1995.

[8] X. Chen, C. Wang, and X. Zhang, "DOA and noncircular phase estimation of noncircular signal via an improved noncircular rotational invariance propagator method," Mathematical Problems in Engineering, vol. 2015, Article ID 235173, 12 pages, 2015.

[9] M. Zhou, X. Zhang, X. Qiu, and C. Wang, “Two-dimensional DOA estimation for uniform rectangular array using reduced-dimension propagator method," International Journal of Antennas and Propagation, vol. 2015, Article ID 485351, 10 pages, 2015.

[10] J. Li, X. Zhang, and H. Chen, "Improved two-dimensional DOA estimation algorithm for two-parallel uniform linear arrays using propagator method," Signal Processing, vol. 92, no. 12, pp. 3032-3038, 2012.

[11] N. Tayem and H. M. Kwon, "Azimuth and elevation angle estimation with no failure and no eigen decomposition," Signal Processing, vol. 86, no. 1, pp. 8-16, 2006.

[12] H. Chen, C. Hou, Q. Wang, L. Huang, W. Yan, and L. Pu, "Improved azimuth/elevation angle estimation algorithm for three-parallel uniform linear arrays," IEEE Antennas and Wireless Propagation Letters, vol. 14, pp. 329-332, 2015.

[13] K. T. Wong and M. D. Zoltowski, "Direction-finding with sparse rectangular dual-size spatial invariance array," IEEE Transactions on Aerospace and Electronic Systems, vol. 34, no. 4, pp. 1320-1336, 1998.

[14] M. D. Zoltowski and K. T. Wong, "Closed-form eigenstructure-based direction finding using arbitrary but identical subarrays on a sparse uniform Cartesian array grid," IEEE Transactions on Signal Processing, vol. 48, no. 8, pp. 2205-2210, 2000. 


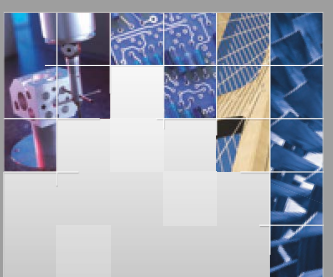

\section{Enfincering}
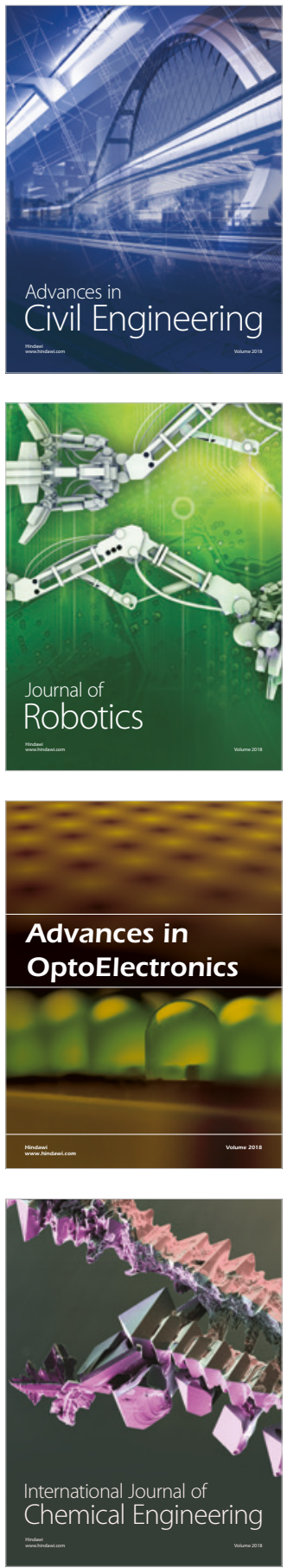

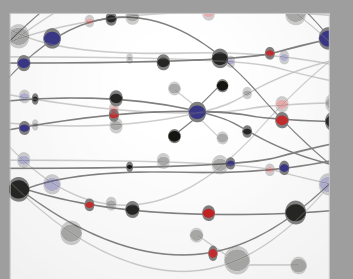

\section{Rotating \\ Machinery}

The Scientific World Journal

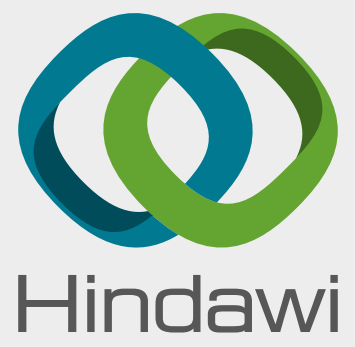

Submit your manuscripts at

www.hindawi.com
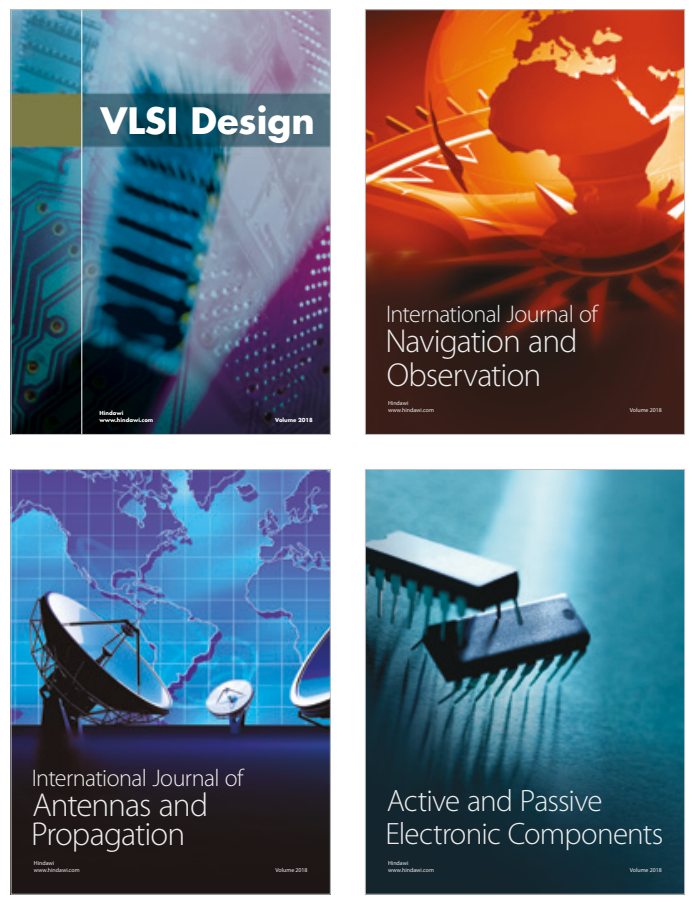
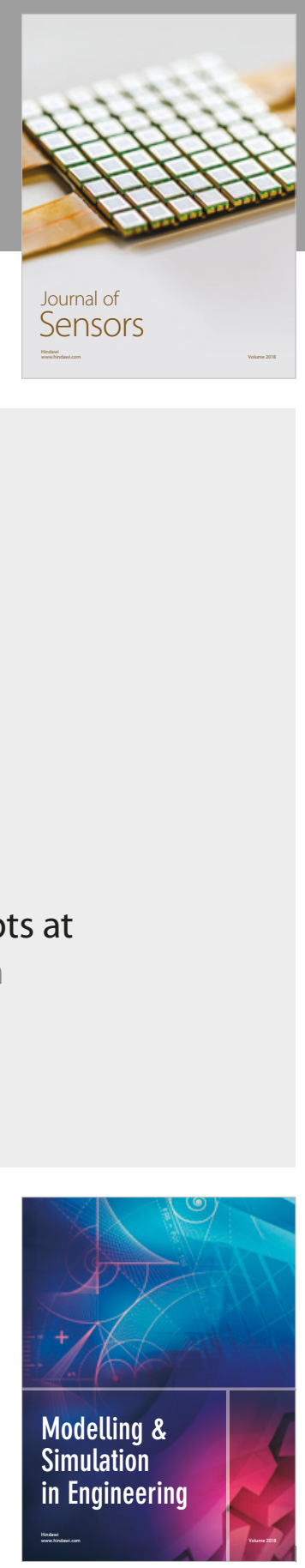

\section{Advances \\ Multimedia}
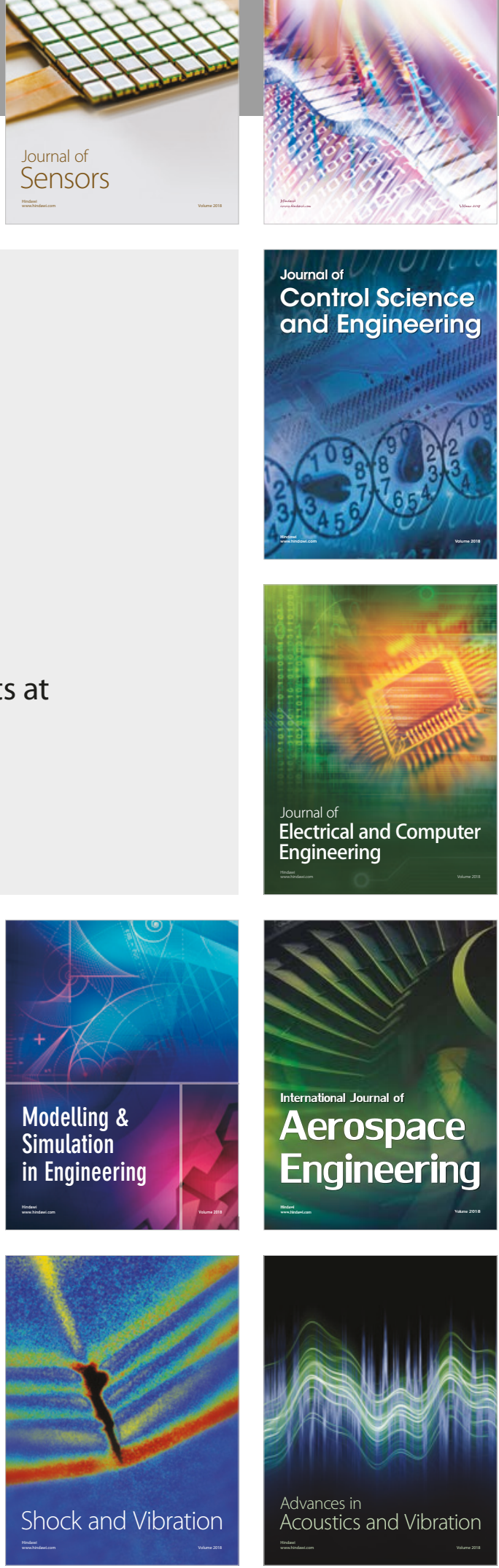